\title{
Methylene Blue removal using a leached graphite prepared by a green mechanochemical process.
}

G. Tarango-Rivero ${ }^{1}$, P. Pizá-Ruíz ${ }^{1}$, C.G. Garay-Reyes ${ }^{2}$, I. Estrada-Guel ${ }^{2}$ and R. Martínez-Sánchez ${ }^{3}$

${ }^{1}$ Centro de Investigación en Materiales Avanzados (CIMAV), Chihuahua, Chihuahua, Mexico, ${ }^{2}$ Centro de Investigación en Materiales Avanzados (CIMAV), Laboratorio Nacional de Nanotecnología, Miguel de Cervantes No. 120, CP 31136, Chihuahua, Chih., México., Chihuahua, Chihuahua, Mexico, ${ }^{3}$ Centro de Investigación en Materiales Avanzados (CIMAV) Laboratorio Nacional de Nanotecnología, Miguel de Cervantes No. 120, CP 31136, Chihuahua, Chih., México., Chihuahua, Chihuahua, Mexico

Methylene blue (MB) is a widely used colorant in hair, paper, cotton, wool, and skin processing. Unfortunately, human exposure to high concentrations can cause health problems which makes this compound a public concern. To treat effluents that have been dye polluted, a variety of physicochemical processes have been used. Despite some satisfactory results obtained, there are serious limitations on their use related to their high costs and low efficiencies [1,2]. Chemisorption can be used as a treatment option; here, the adsorbate (dye) is retained on an adsorbent solid surface and inner pores [3]. This work presents an efficient alternative for AM absorption in an aqueous solution, using natural (Gr) and leached graphite (GrL). GrL was prepared following a mechanochemical route based on milling of an equimolar mixture of Gr flakes and calcium carbonate in a Spex mill for 16 hours; the milled powders were cold leached with acetic acid, washed, and dried. AM removal testing was carried out, adding $100 \mathrm{~mL}$ of an aqueous dye solution $(30 \mathrm{mg} / \mathrm{L})$ and $20 \mathrm{mg}$ of graphite in an agitated glass beaker at room temperature. The adsorption studies were performed during 1 hour with 10 min intervals taking a centrifugated sample of $5 \mathrm{~mL}$ and reading it using a Cary 5000 UV-Vis-NIR spectrophotometer and calculating the dye removal percentage.

In Fig. 1, it is evident that the original Gr has a large particle size with a flaky morphology; after the mechanochemical processing, there is notable powder comminution and functionalization with oxygenbased radicals (oxygen content varies from 0 to 17\%) and full calcium lixiviation (from calcium carbonate milling), at $50 \mathrm{KX}$ magnification, the sample shows a high defoliation level and homogeneity. As shown in Fig. 2, these characteristics increase the MB removal rate of the GrL sample considerably. The peak adsorption was 98\%; meanwhile, the original Gr showed no effect on the MB concentration. This increased removal efficiency of GrL sample could be related to its small particle size, high exfoliation level, and oxygen concentration. Removal rate variation is a function of time. The longer the time, the greater the MB removal. 



Figure 1. SEM images obtained through a JSM-7401F microscope with their corresponding elemental composition and $50 \mathrm{KX}$ magnification of original (a, c) and leached (b, d) samples.



Figure 2. MB removal efficiently curves as a function of time.

\section{References}

[1] S. Wang, H. Li. Kinetic modelling and mechanism of dye adsorption on unburned carbon, Dyes and Pigments 72-3 (2007) 308-314.

[2] E. Eren, B. Afsin. Investigation of a basic dye adsorption from aqueous solution onto raw and pretreated sepiolite surfaces, Dyes and Pigments 73 (2007) 162-167.

[3] S. Wang, Z.H. Zhu. Effects of acidic treatment of activated carbons on dye adsorption, Dyes and Pigments 75 (2007) 306-314. 